\title{
Formación y atención a la diversidad funcional desde la Universidad: percepciones del alumnado universitario
}

\section{Training and attention to functional diversity from the University: perceptions of university students}

\author{
Carmen $\mathrm{M}^{\mathrm{a}}$ Hernández Garre, Borja Avilés Soler \\ Universidad de Almería, España
}

\begin{abstract}
Resumen
La atención a la diversidad funcional en el ámbito de la Educación Superior supone uno de los retos más complejos ante la inclusión socioeducativa. La institución debe proporcionar un currículo adaptado, una enseñanza individualizada y una formación inicial y continua de los futuros profesionales. Así, se aplicó un cuestionario sobre ideas y actitudes ante la integración educativa y social de los estudiantes con discapacidad con 92 estudiantes del Grado en Psicología en la Universidad de Minho (Portugal). Como resultados más relevantes se ponía de relieve la necesidad de una mejora en las adaptaciones metodológicas y en la infraestructura de la Universidad. Palabras clave: Educación superior, educación inclusiva, necesidades educativas especiales.
\end{abstract}

\begin{abstract}
English abstract. Attention to functional diversity in the field of Higher Education is one of the most complex challenges to socio-educational inclusion. The institution should provide an adapted curriculum, individualized instruction and initial and continuing training of future professionals. Thus, a questionnaire on ideas and attitudes was applied to the educational and social integration of students with disabilities with 92 students of the Degree in Psychology at the University of Minho (Portugal). The most relevant results highlighted the need for an improvement in the methodological adaptations and in the infrastructure of the University.

Keywords: Higher education, inclusive education, special educational needs.
\end{abstract}

\section{Objetivos}

Atendiendo a la relevancia que supone la inclusión educativa y social de los estudiantes con discapacidad en el contexto universitario, desde una perspectiva de formación integral a través de una educación basada en la adquisición de competencias, se ha planteado el desarrollo de este estudio con el principal propósito de conocer las ideas que el alumnado posee sobre dicha temática. Así, los objetivos concretos de la investigación son:
- Analizar las opiniones sobre la integración de los estudiantes con discapacidad en la universidad de Minho (Braga, Portugal).

- Examinar de las ideas sobre dificultades y necesidades relacionadas con la formación integral de los estudiantes con discapacidad.

\section{Método}

\section{Participantes}

A través de un muestreo aleatorio simple entre los estudiantes de primero de la titulación de Grado en Psicología se seleccionó una muestra de 92 estudiantes de la Universidad de Minho que participaron en el estudio.

\section{Instrumentos}

El instrumento utilizado para la recogida de información sobre las percepciones del alumnado fue una encuesta que fue adaptada a partir del cuestionario sobre ideas y actitudes ante la integración educativa y social de los estudiantes con discapacidad, siendo extraída de Sánchez (2009), en función de las necesidades concretas del contexto portugués.

\section{Procedimiento}

Teniendo en cuenta los objetivos planteados, en esta investigación se ha adoptado un diseño metodológico de tipo descriptivo, utilizando como técnica de investigación la encuesta, una técnica que permite la obtención de datos a través del cuestionario para el análisis descriptivo-cuantitativo e interpretación de resultados (Goetz y LeCompte, 1988).

Tras la obtención de datos a través del instrumento utilizado, se ha llevado a cabo el análisis descriptivo de la percepción del alumnado en relación con la integración de los estudiantes con discapacidad en el contexto universitario, utilizando para ello estadísticos tales como la frecuencia $y$ el porcentaje, junto con los correspondientes gráficos. Finalmente, atendiendo a los datos analizados se ha reflexionado acerca de los obstáculos y retos que presenta en la actualidad la inclusión educativa y social en la universidad de Minho, 
estableciendo algunas pautas imprescindibles para la mejora de la formación de los estudiantes con discapacidad.

\section{Resultados}

Se presentan a continuación los resultados más relevantes obtenidos en el cuestionario mencionado sobre ideas y actitudes relacionadas con la integración educativa y social de los estudiantes con discapacidad en la Universidad de Minho:

En relación al fomento de actitudes favorables hacia la integración de estudiantes con discapacidad, el 46,3\% de los estudiantes se mostraba en desacuerdo con dicha afirmación.

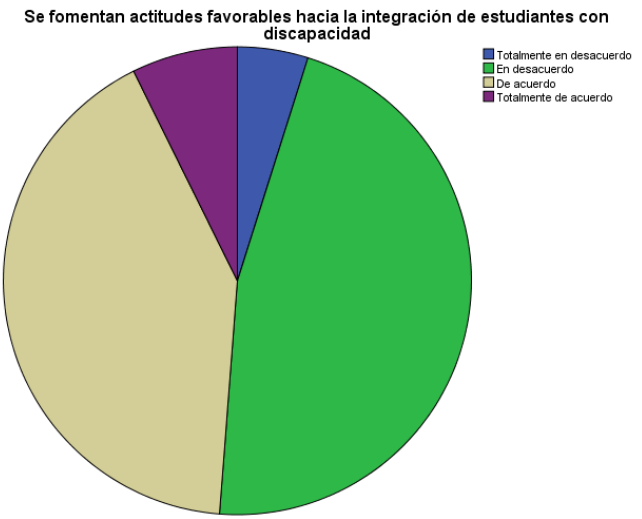

Figura 1. Fomento de actitudes favorables hacia la integración de estudiantes con discapacidad.

Según el 58,5 \% de los estudiantes afirmaba no haber recibido algún asesoramiento o formación para facilitar su estancia en la universidad

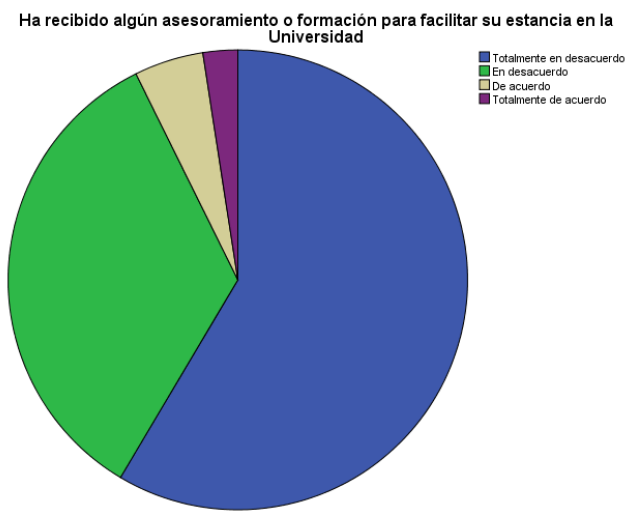

Figura 2. Ha recibido algún asesoramiento o formación para facilitar su estancia en la universidad.

En relación a si la metodología (espacios, mobiliario, materiales, horarios, etc.) consideran la atención a la diversidad por discapacidad, un 56,1 \% mostraba la necesidad de mejoras en ese sentido.

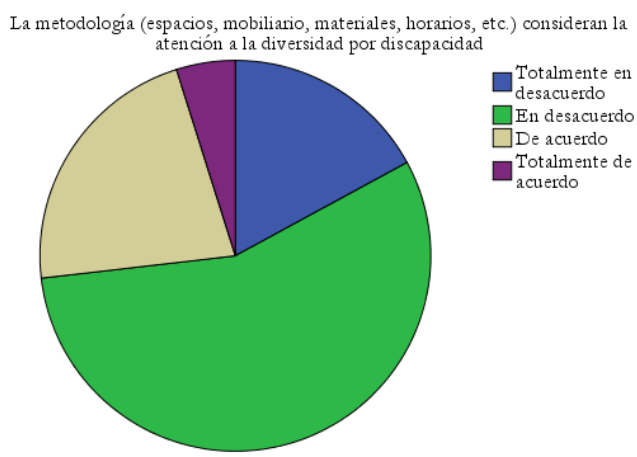

Figura 3. La metodología considera la atención a la diversidad

En cuanto a la existencia de adaptaciones de la infraestructura de la Universidad para las diversas discapacidades, un $48 \%$ de los estudiantes se manifestaron en desacuerdo y totalmente en desacuerdo $(17 \%)$.

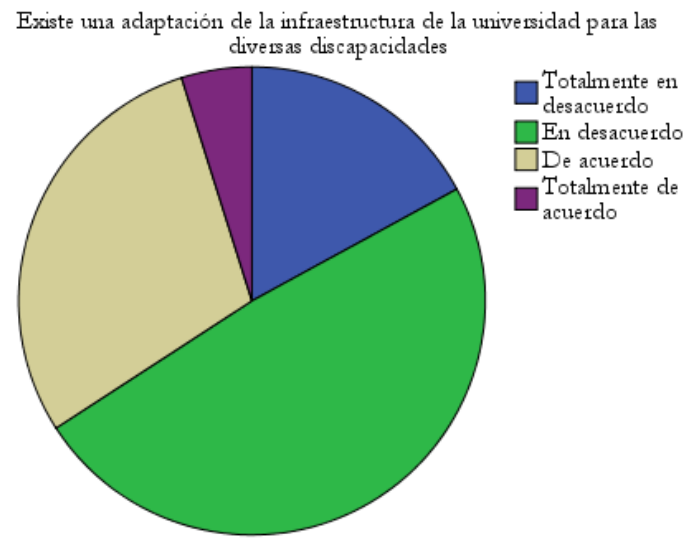

Figura 4. Existe una adaptación de la infraestructura de la universidad para las diversas discapacidades.

Un $63,4 \%$ de los participantes en el estudio se mostraba en desacuerdo con que existiera en la universidad un Plan de Acogida para los estudiantes con discapacidad.

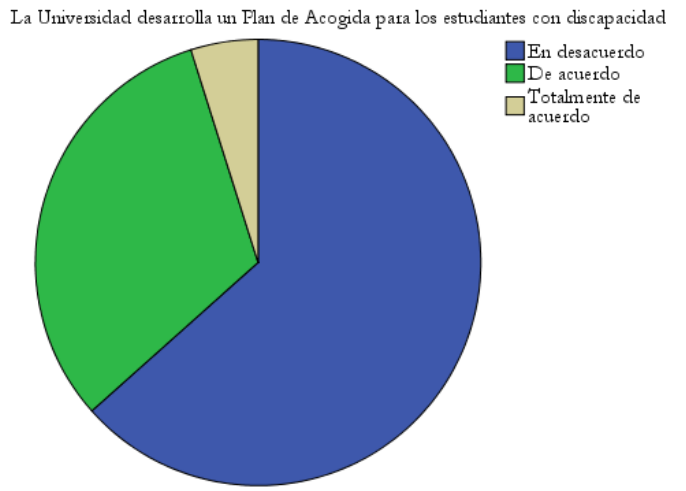

Figura 5. La universidad desarrolla un Plan de Acogida para los estudiantes con discapacidad. 
En cuanto a la existencia en la universidad de profesorado especializado para la atención a los estudiantes con discapacidad, un $63,4 \%$ se mostraba en desacuerdo y un $24,4 \%$ de acuerdo.

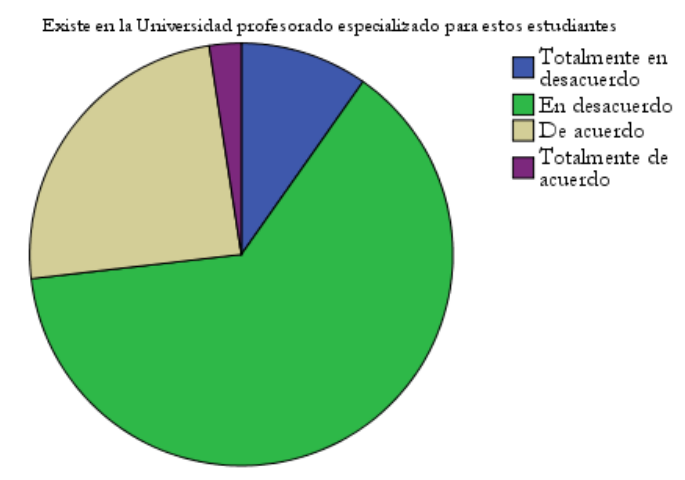

Figura 6. Unidad de profesorado especializado para estudiantes con discapacidad.

Cuando se les preguntaba si la presencia en las aulas de estudiantes con discapacidad suponía problemas o dificultades, la mayoría de los participantes $(48,8 \%)$ se mostraba en total desacuerdo. En cuanto a la comparación entre las oportunidades o posibilidades de promoción de los estos estudiantes, un $48,8 \%$ señalaba que estaba de acuerdo con que tenían igualdad de oportunidades.

En relación a la percepción sobre el fomento del cumplimiento de normativas universitarias que determinan los derechos y deberes de las personas con discapacidad, el 58,5\% se mostraba de acuerdo y totalmente de acuerdo $(9,8)$. Sin embargo, cuando se les preguntaba sobre si se promueve la concienciación de la comunidad educativa acerca de los servicios disponibles para los alumnos con discapacidad, el $46,3 \%$ y el 7,3\% se mostraba en desacuerdo y totalmente desacuerdo respectivamente.

\section{Conclusiones}

El ingreso de estudiantes con discapacidad en la Enseñanza Superior es una realidad cada vez más presente en nuestro entorno que supone un reto importante en los sistemas universitarios. Así, a pesar de ser muchos los avances con respecto a este proceso, el análisis de la realidad muestra diferencias con respecto a la información, la incorporación y la atención sobre las condiciones especiales en las que se desenvuelven los estudiantes con necesidades. En el presente trabajo se evidencia igualmente la necesidad de un conocimiento previo de esas necesidades, tanto por parte de la institución, como por parte de los docentes que permitan diseñar estrategias reales para la inclusión en la Educación Superior. La necesidad de reforzar diferentes aspectos relacionados con la institución universitaria para la incorporación y permanencia de estudiantes con necesidades, queda patente en este estudio. No obstante, a pesar de las dificultades señaladas, es importante apuntar que un alto porcentaje de los participantes en el estudio percibe un adecuado cumplimiento de la normativa universitaria relacionada con el respeto de los derechos y deberes de las personas con discapacidad. Por otro lado, se manifiesta la necesidad de formación que promueva unos principios inclusivos, máxime en los estudiantes que se dediquen en un futuro a la tarea educativa y psicológica Para ello se requiere una formación inicial y continua del profesorado basada en conocimientos, competencias, valores y actitudes, vinculándose todos estos elementos al principio de justicia social y alejándose del modelo del déficit.

Esta formación resulta de vital importancia para el desarrollo de la inclusión en la sociedad por parte de los futuros educadores (Watkins, 2009). Concretamente, con relación a las actitudes, se sabe que el profesorado que posee una predisposición más favorable hacia la inclusión de alumnado con necesidades educativas especiales (NEE), es aquel que también dispone de más estrategias educativas para que la inclusión tenga éxito (Ahmmed, Sharma y Deppeler, 2012; Avramidis y Norwich, 2002; Chiner, 2011; Swain, Nordness y Leader-Janssen, 2012). En Portugal es notorio el creciente número de alumnos con NEE que entraron en el Educación Superior en los últimos años, pero todavía existe una población muy reducida si se compara con el número total de estudiantes que ingresan en la Universidad. Para García, Rodriguero y Mori (2011), estos resultados son resultado de la segregación, la extinción y la discriminación que sufren las personas con necesidades especiales a lo largo de la historia. Se observa que la paradoja de la inclusión frente a la exclusión en los sistemas educativos universaliza el acceso a los mismos, pero la exclusión pasa factura a las personas y grupos que no encajan en la homogeneización de las normas establecidas por este sistema educativo (García, Rodriguero, y Mori, 2011). En esta línea existen trabajos precedentes al que aquí se presenta como el que coordinó el doctor Sánchez Palomino en la Universidad de Almería, el cual estudia un problema con una incidencia cada día mayor en la Universidad: la atención a los estudiantes con discapacidad.

\section{Referencias}

Ahmmed, M., Sharma, U. y Deppeler, J. (2012). Variables affecting teachers' attitudes towards inclusive education in Bangladesh. Journal of Research in Special Education Needs, 12 (3), 132-140.

Avramidis, E., Bayliss, P. y Burden, R. (2000). A survey into mainstream teachers' attitudes towards the inclusion of children with special educational needs in the ordinary school in one local authority. Educational Psychology, 20 (2), 191- 211.

Chiner, E. (2011). Las percepciones y actitudes del profesorado hacia la inclusión del alumnado con necesidades educativas especiales como indicadores del uso de prácticas educativas inclusivas en el aula. Tesis doctoral. Universidad de Alicante.

García, D., Rodriguero, C., y Mori, N. (2011). Inclusão no Ensino Superior: O olhar do aluno. In Atas do VII Encontro da Associação Brasileira de Pesquisadores em Educação Especial (pp. 574-583). Londrina: UEL. 
Goetz, J. P. y LeCompte, M. D. (1988). Etnografia y diseño cualitativo en investigación educativa. Madrid: Ediciones Morata.

Sánchez, A. (2009). La integración educativa y social de los estudiantes con discapacidad en la universidad de Almería. Almería: Universidad de Almería.

Swain, K. D., Nordness. P. D. y Leader-Janssen, E. M. (2012). Changes in preservice teacher attitudes toward inclusion. Preventing School Failure, 56 (2), 75- 81.

Watkins, A. (ed.) (2009). Special Needs Education Country Data 2008. Odense: Agencia Europea para el Desarrollo de la Educación del Alumnado con Necesidades Educativas Especiales. 